\title{
Degenerate topological line surface phonons in quasi-1D double helix crystal SnIP
}

\author{
Bo Peng $\mathbb{D}^{1}$, Shuichi Murakami $\mathbb{D}^{2,3}$, Bartomeu Monserrat ${ }^{1,4 凶}$ and Tiantian Zhang $\mathbb{D}^{2,3 凶}$
}

Degenerate points/lines in the band structures of crystals have become a staple of the growing number of topological materials. The bulk-boundary correspondence provides a relation between bulk topology and surface states. While line degeneracies of bulk excitations have been extensively characterised, line degeneracies of surface states are not well understood. We show that SnIP, a quasi-one-dimensional van der Waals material with a double helix crystal structure, exhibits topological nodal rings/lines in both the bulk phonon modes and their corresponding surface states. Using a combination of first-principles calculations, symmetrybased indicator theories and Zak phase analysis, we find that two neighbouring bulk nodal rings form doubly degenerate lines in their drumhead-like surface states, which are protected by the combination of time-reversal symmetry $\mathcal{T}$ and glide mirror symmetry $\bar{M}_{y}$. Our results indicate that surface degeneracies can be generically protected by symmetries such as $\mathcal{T} \bar{M}_{y}$, and phonons provide an ideal platform to explore such degeneracies.

npj Computational Materials (2021)7:195; https://doi.org/10.1038/s41524-021-00667-6

\section{INTRODUCTION}

Degeneracies in the bulk energy bands of crystals were intensively studied in the early days of band theory ${ }^{1}$. Recent breakthroughs have shed new light into this old topic by associating topological invariants with these degenerate points or lines ${ }^{2-4}$, and by extending the discussion from electrons to phonons ${ }^{5-7}$, photons ${ }^{8}$, magnons $s^{9}$ and excitons ${ }^{10}$. Among the degenerate points, twofold degenerate Weyl points always appear in pairs that can be topologically characterised with opposite Chern numbers ${ }^{11-14}$, and fourfold degenerate Dirac points have a linear dispersion in both electronic ${ }^{15-20}$ and phononic systems ${ }^{21,22}$. When the band crossings are one-dimensional in momentum space, they can form nodal lines, nodal rings or nodal chains, depending on their shape ${ }^{23-31}$, and these line crossings are protected by symmetries such as mirror or $\mathcal{P} \mathcal{T}$ (where $\mathcal{P}$ is the spatial inversion symmetry and $\mathcal{T}$ is the time-reversal symmetry ${ }^{32,33}$. Recent advances, using both group-theoretical analysis and high-throughput calculations, have enabled a comprehensive understanding of bulk band degeneracies in both electrons ${ }^{34-36}$ and phonons ${ }^{22}$. These topological degeneracies exhibit unique physical properties such as a 'quantum anomaly'37,38, enabling various applications from spintronics $^{39}$ to topological quantum computation ${ }^{40}$.

As a result of the bulk-boundary correspondence, the degenerate bulk points and lines are associated with topologically protected surface states, including surface arcs associated with Weyl and Dirac points ${ }^{41-44}$ and drumhead-like flat bands associated with nodal lines ${ }^{24,45}$. Similar to bulk band degeneracies, surface states can also exhibit band crossings in both semi-infinite slabs and finite slabs (with top and bottom surfaces) ${ }^{46}$. Here we focus on semi-infinite systems with only one surface. For Weyl points with Chern numbers of \pm 1 , the associated helicoid surface states, whose isoenergy contours are Fermi arcs, form a continuous 2D surface in the energy-momentum space $E-\mathbf{k}$ and have no degeneracy ${ }^{47}$, as schematically shown in Fig. 1a. In topological insulators and topological crystalline insulators, the surface states become degenerate at a single point ${ }^{48-52}$, forming a surface Dirac cone, as shown schematically in Fig. 1b. In principle, two surface states can also cross along a line in the surface Brillouin zone, as shown schematically in Fig. 1c. However, like the bulk case, these surface line crossings need to be protected by additional symmetries ${ }^{47,53-56}$. While nodal line degeneracies in the bulk states have been thoroughly explored in a variety of quasiparticle band structures, the degenerate lines in the surface states have received less attention. The few examples of surface nodal lines all correspond to electronic band structures ${ }^{47,53-57}$ or artificial systems ${ }^{58,59}$. It is therefore desirable to identify more candidates in other quasiparticle spectra to fully understand the role of surface degeneracies.

Among all the quasiparticles, phonons are of particular interest as they describe intrinsic ionic vibrations. Moreover, the bosonic nature of phonons facilitates the exploration of topological phenomena in their entire spectrum. This is in contrast to fermionic systems (e.g. electronic systems) in which there is an additional constraint that degeneracies need to occur near the Fermi level to have an impact on the behaviour of the material. Furthermore, time-reversal symmetry is hard to break in phononic systems, and consequently, this extra symmetry requirement is automatically satisfied in phonons. These advantages have led to a recent surge of interest in characterising topological properties of phonons ${ }^{5-7,21,22,60-76}$, but degenerate lines in surface phonon states have not yet been reported.

In this work, we propose that SnIP, an inorganic semiconductor with nodal-ring phonons in the bulk states, exhibits nodal-line surface states in the phonon spectrum using a combination of first-principles calculations, group theory analysis and Zak phase analysis. These surface states are doubly degenerate in a nodal line on the (100) surface. The surface nodal line is protected by the anti-unitary symmetry $\vartheta=\mathcal{T} \bar{M}_{y}$, which is a combination of

\footnotetext{
${ }^{1}$ TCM Group, Cavendish Laboratory, University of Cambridge, J. J. Thomson Avenue, Cambridge CB3 OHE, United Kingdom. ${ }^{2}$ Department of Physics, Tokyo Institute of Technology, Ookayama, Meguro-ku, Tokyo 152-8551, Japan. ${ }^{3}$ Tokodai Institute for Element Strategy, Tokyo Institute of Technology, Nagatsuta, Midori-ku, Yokohama, Kanagawa 226-8503, Japan. ${ }^{4}$ Department of Materials Science and Metallurgy, University of Cambridge, 27 Charles Babbage Road, Cambridge CB3 0FS, United Kingdom.

凶email: bm418@cam.ac.uk; ttzhang@stat.phys.titech.ac.jp
} 
(a)

\section{helicoid surface states}

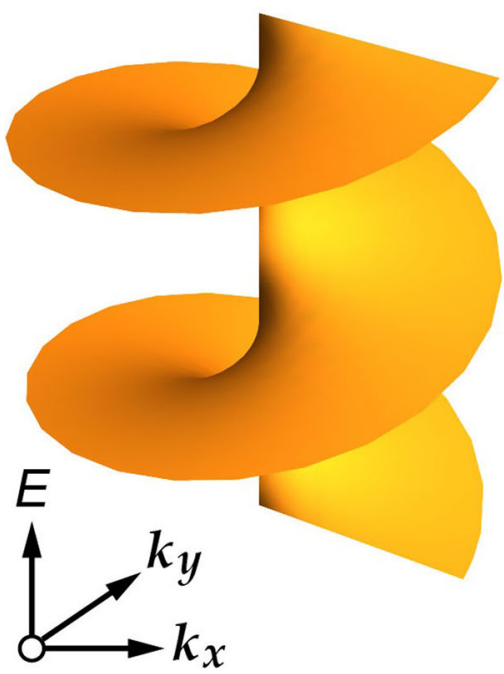

(b)

Dirac surface states

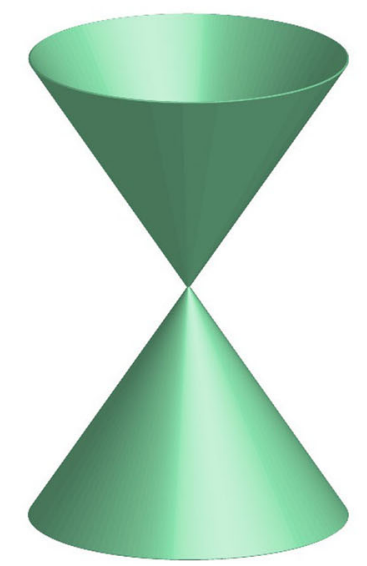

(c)

\section{nodal-line surface states}

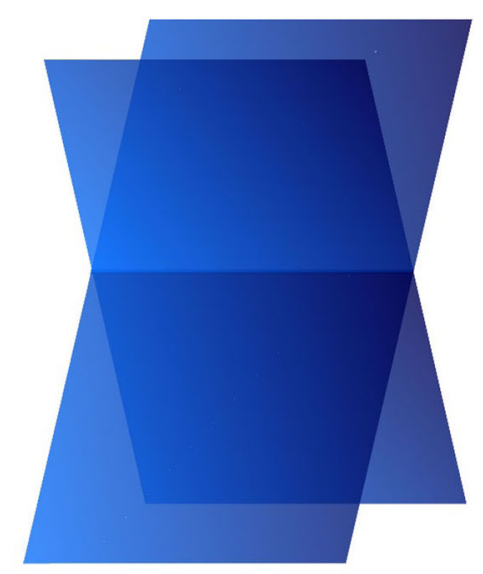

Fig. 1 Different types of surface states in topological materials. a Nondegenerate helicoid surface states, b zero-dimensional degenerate Dirac surface states and c one-dimensional degenerate nodal-line surface states.

time-reversal symmetry $\mathcal{T}$ and glide mirror symmetry $\bar{M}_{y}$. With different surface terminations, we can alternatively get two surface nodal lines on different parts of the (100) surface Brillouin zone, which can be understood by different Zak phases in the presence of $\mathcal{T} \bar{M}_{y}$ symmetry. Our results suggest that similar surface degenerate lines/points will be found in other materials when there are extra symmetries to protect these surface degeneracies, and we believe that phonons can be a primary platform to study these degeneracies because of the presence of time-reversal symmetry and the flexibility to study the degeneracies in the entire phonon spectrum.

\section{RESULTS}

\section{Crystal structure and phonon dispersion}

SnIP is an inorganic semiconductor with a double helix crystal structure, first synthesised in 2016, and can be formed without any templates ${ }^{77-79}$. The strong covalent bonds along the helices and the weak van der Waals interactions across the helices combine the electronic properties of inorganic semiconductors, such as high electron mobility ${ }^{80}$, and the mechanical properties of polymers, such as high flexibility ${ }^{81}$. In addition, by atomic substitution, a large material family with double helix structures has been predicted using first-principles calculations, providing a wide range of band gaps between 1 and $2.5 \mathrm{eV}^{82}$. With a tunable bandgap and high mechanical flexibility, these quasi-one-dimensional semiconductors are extremely promising for next-generation devices ranging from mechanical sensors to optoelectronics ${ }^{81,82}$. Moreover, they can serve as a material platform to realise one-dimensional physical models such as the Su-Schrieffer-Heeger model ${ }^{83}$ and Majorana quantum wires ${ }^{84,85}$.

Bulk SnIP crystallises in the monoclinic space group $P 2 / C$ (No. 13) with two double helices $(S n I P)_{7}$ in the unit cell with stoichiometry $(\mathrm{SnIP})_{14}$. The two double helices, alternatively leftand right-hand twisted, are stacked along the $a$ direction. Each double helix is formed by an inner helical $P$ chain and an outer helical Snl chain, as shown in Fig. 2a. The $(S n I P)_{7}$ unit in each SnIP chain winding results in a $7 / 2$ helix. From one phosphorus (yellow) atom to the next, the turning angle is about $\pm 360^{\circ} / 7$ followed by a translation of $c / 7$, and the same holds true for the tin (cyan) and iodine (navy) atoms in the Snl helix ${ }^{86}$. The calculated lattice parameters of $a=7.889 \AA, b=9.768 \AA, c=$ $18.422 \AA$, and monoclinic distortion $\beta=110.21^{\circ}$ match well with the measured ones ${ }^{77,79}$.

The calculated phonon dispersion of SnIP is presented in Fig. 2b. It exhibits wide bandwidths along the helical direction, corresponding to the $\Gamma-Y, C-Z, B-A$ and $E-D$ high-symmetry lines in Fig. 2c, but, by contrast, phonon bands along other highsymmetry lines, especially those perpendicular to the helices, have a relatively flat dispersion, a result of the quasi-onedimensional nature of this material. This is consistent with the fact that SnIP has strong covalent bonds along the onedimensional helices but only weak van der Waals-like interactions between the double helices. As a result, SnIP fibres can be macroscopically bent by up to $90^{\circ}$ without any significant Raman mode shifts, indicating that SnIP is an ultrasoft inorganic material and bending the helices has negligible influence on the phononic properties ${ }^{81}$.

There are 42 atoms in the unit cell of SnIP, corresponding to 126 phonon branches. The calculated atom-projected phonon density of states (DOS) in Fig. $2 \mathrm{~b}$ indicates that the low-frequency modes below $4 \mathrm{THz}$ are mainly vibrations involving the heavier $\mathrm{Sn}$ and I atoms, while those between 4-14 THz are dominated by the motion of the lighter $\mathrm{P}$ atoms. There are 14 phosphorus atoms in the unit cell, leading to $42 \mathrm{P}$-dominated bands in the phonon spectra, spanning branches 85 to 126 . The phonon modes from 4 to $10 \mathrm{THz}$ (branches $85-108$ ) are more dispersive compared to the almost flat bands between 10 and $14 \mathrm{THz}$. The two double helices are related to each other by inversion/glide mirror symmetry, so there always exist two bands with similar eigenvalues and similar eigenvectors, corresponding to the $P$ atoms from the two separate $P$ chains, respectively (see the phonon dispersion between $4-8.5 \mathrm{THz}$ in Supplementary Note 1 of the Supplementary Information).

Hereafter we focus on the frequency range 4-10 THz because the corresponding phonon bands are relatively 'clean' due to the large mass difference (between phosphorus and the other two elements) that separates them from the lower energy bands, and 
(a)

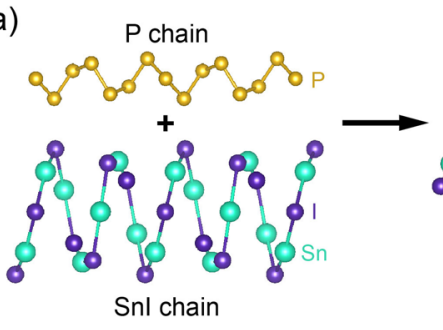

(b)

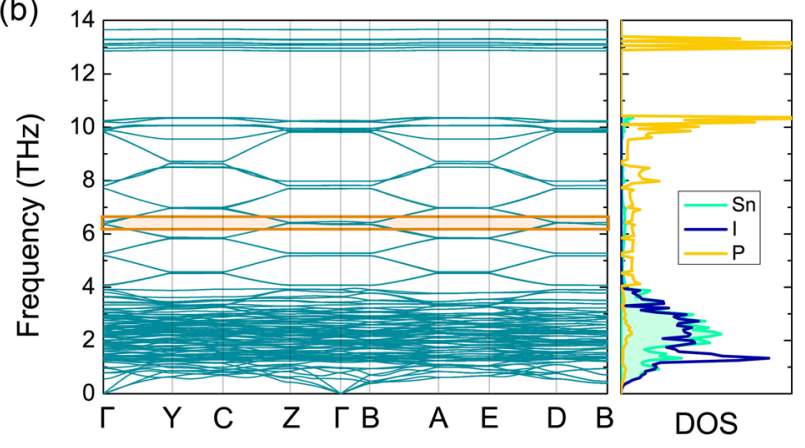

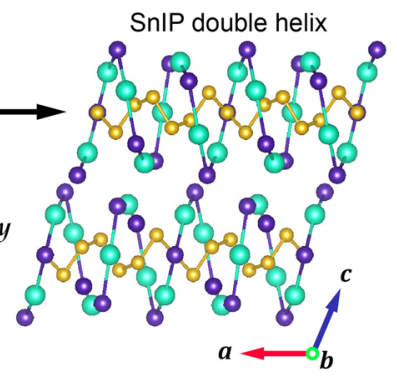
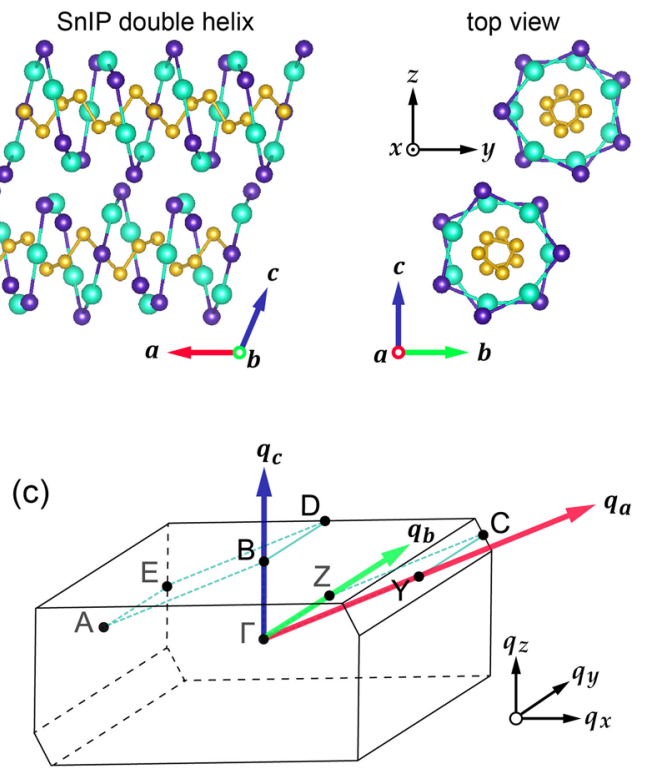

Fig. 2 Structural and vibrational properties of SnIP. a Crystal structure, b phonon dispersion and c Brillouin zone of SnIP. The orange region in the phonon dispersion highlighted in (b) corresponds to the topological features that we focus on. The phonon DOS is also shown in (b).

due to the strong intrachain vibrations that make them highly dispersive. This makes them an ideal playground for exploring topological features. In addition, these modes in the range 4-10 THz contribute to nearly half the thermal conductivity along the helix direction (see the phonon transport properties in Supplementary Note 2 of the Supplementary Information).

\section{Nodal-ring phonons in SnIP}

For the phonon modes between 4 and $10 \mathrm{THz}$, dominated by the vibrations of the relatively light $P$ atoms, the phonon bands have a large dispersion along the helical direction but are almost flat along with the perpendicular directions, as shown in Fig. $2 \mathrm{~b}$. The band folding of the seven $P$ atoms in a single helical $P$ chain ${ }^{86}$ leads to several band crossings near the Brillouin zone boundary, especially in the flat band regions. After a comprehensive search for band crossing points between bands $85-108$ (i.e. 4-10 THz), we find that the most promising band crossings are formed by phonon branches 92 and 93 with clean topological features in both bulk and surface states. Marked with \# 92 and \# 93 in Fig. 3b, these two bands cross along the $\Gamma-Z$ high-symmetry line.

To understand the topological information of the band crossings between branches 92 and 93, we use symmetry-based indicator theories to analyze the degeneracies ${ }^{63,87-91}$. Because of the existence of a band crossing along the $\Gamma-Z$ high-symmetry line, the compatibility condition is broken for the lowest 92 bands, as the compatibility relations can only be satisfied when the band structures have no (non-accidental) band crossings along any high-symmetry lines or at any high-symmetry points ${ }^{88}$. So we cannot use the symmetry-based indicator for space group No. 13. Instead, we need to find a maximal subgroup of SnIP that simultaneously satisfies the compatibility condition and has a nontrivial symmetry-based indicator. Among all the subgroups of space group No. 13 (space groups No. 2, No. 3 and No. 7), No. 2 is the only subgroup, which both satisfies the compatibility condition and has a symmetry-based indicator group: (i) the compatibility condition is satisfied because the twofold rotation symmetry in No. 13 that protects the band crossing along $\Gamma-Z$ is absent in No. 2; (ii) space group No. 2 has a nontrivial symmetrybased indicator of $\mathbb{Z}_{2224} \equiv \mathbb{Z}_{2} \times \mathbb{Z}_{2} \times \mathbb{Z}_{2} \times \mathbb{Z}_{4}$, as discussed in detail in ref. ${ }^{88}$. Therefore we can use $\mathbb{Z}_{2224}$ as indicators to diagnose topological degeneracy. For space group No. 2, there are eight high-symmetry points, i.e. high-symmetry points $\left(q_{a}, q_{b}, q_{c}\right)$ with $q_{a, b, c}=0,0.5$. We obtain the irreducible representations (irreps.) at the eight corresponding high-symmetry points $\Gamma(0,0,0)$, Z $(0,0.5,0)$, B $(0,0,0.5), Y(0.5,0,0), C(0.5,0.5,0), D(0,0.5,0.5), A$ $(0.5,0,0.5)$ and $E(0.5,0.5,0.5)$, as shown in Fig. 3a. We then compute the indicator accordingly. The resulting indicator (0101) implies that there is a single nodal ring around the $Z$ point in SnIP formed by branches 92 and 93, which is verified by the numerical calculations in Fig. 3c.

We next examine in detail the nodal rings formed by bands 92 and 93 . We zoom in the phonon dispersion on the $\Gamma-B-D-Z$ plane in Fig. $3 \mathrm{~b}$. The band crossing formed by bands 92 and 93 along the $\Gamma-Z$ high-symmetry line belongs to the single nodal ring around the $Z$ point, carrying a quantised $\pi$ Berry phase. The nodal ring crosses through the Brillouin zone boundary around the B-D high-symmetry line, and consequently, there are two nodal rings from two neighbouring Brillouin zones formed around the $D$ point. We also plot the phonon dispersion around $\mathrm{N}(-0.01,0.35$, 0.51), another band crossing point on the neighbouring nodal ring, as shown in Fig. 3b. Most interestingly, we find that the two neighbouring nodal rings projected on the (100) surface have a large overlap, and the two drumhead-like surface states of the two bulk nodal rings form a doubly degenerate line along $\bar{B}-\bar{D}$ (as discussed below).

\section{Topological surface states of SnIP}

We demonstrate the nodal-line surface states by calculating the surface local density of states (LDOS) from the imaginary part of the surface Green's function ${ }^{92}$. We first study the surface along the (100) direction with the termination denoted as surface 1 in Fig. 4a-e. As shown in Fig. $4 a$, along the $\bar{D}-\bar{Z}$ and $\bar{Z}-\bar{\Gamma}$ highsymmetry lines, two separate projections of the bulk band crossings (marked by navy and cyan arrows) belong to two single nodal rings from two neighbouring Brillouin zones respectively. The surface states are within the projections of the two bulk nodal rings, forming two nondegenerate drumhead-like surface states marked by navy and cyan triangles in Fig. 4a respectively. The surface states from the two crossing points along $\bar{D}-\bar{Z}$ and $\bar{Z}-\bar{\Gamma}$ merge with each other at $\bar{D}$, and result in a doubly degenerate 
(a)

\begin{tabular}{cc} 
& No. $13(P 2 / C)$ \\
\hline q point & irreps. \\
\hline$\Gamma$ & $22 \Gamma_{1+}+22 \Gamma_{1-}+24 \Gamma_{2+}+24 \Gamma_{2-}$ \\
$Z$ & $22 Z_{1+}+21 Z_{1-}+25 Z_{2+}+24 Z_{2-}$ \\
B & $46 B_{1}$ \\
$Y$ & $23 Y_{1+}+23 Y_{1-}+23 Y_{2+}+23 Y_{2}$ \\
$C$ & $23 C_{1+}+23 C_{1-}+23 C_{2+}+23 C_{2}$ \\
$D$ & $46 D_{1}$ \\
A & $46 A_{1}$ \\
E & $46 E_{1}$ \\
\hline
\end{tabular}

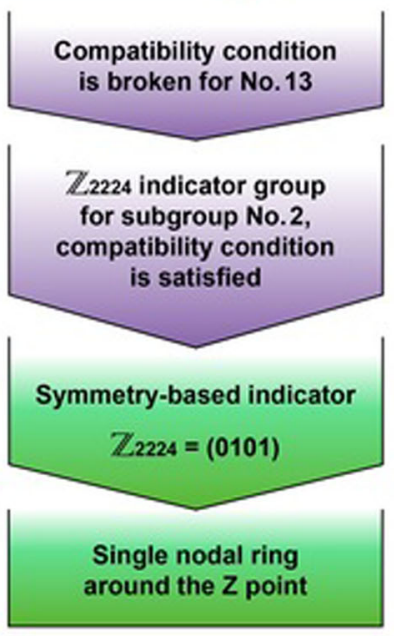

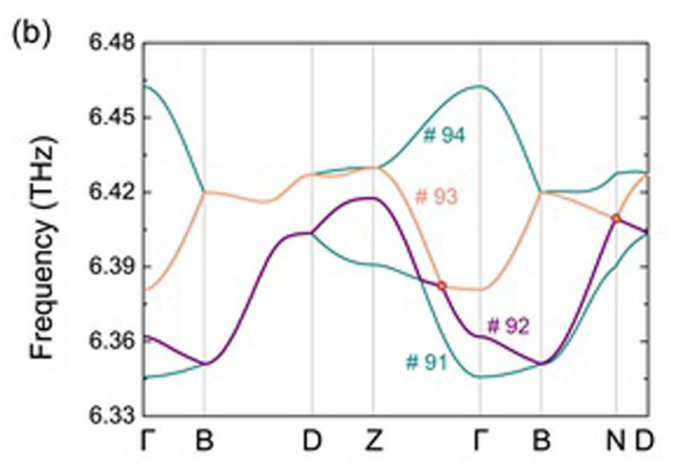

(c)

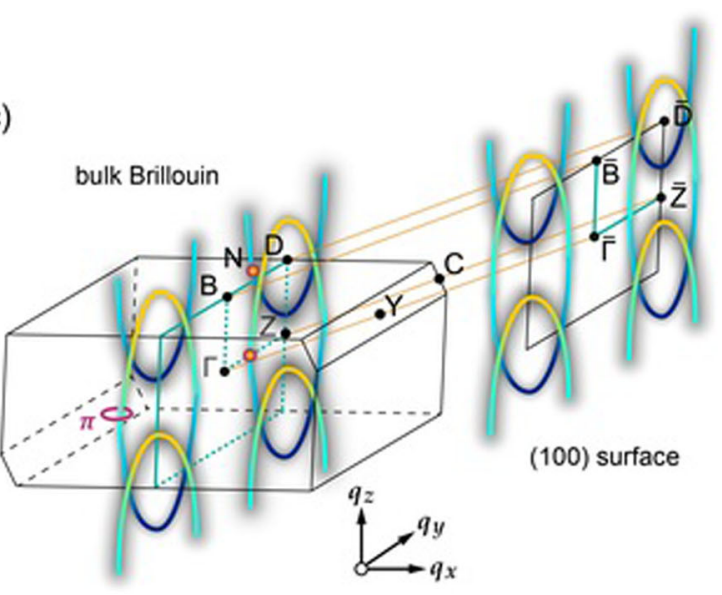

Fig. 3 Topological properties of SnIP. a Diagnosis process for the single nodal ring around the Z point using symmetry-based indicator theory for the lowest 92 bands. b Phonon band crossings between branches 92 and 93 and $\mathbf{c}$ the nodal rings in the bulk Brillouin zone, as well as their projections on the (100) surface.

surface nodal line along $\bar{B}-\bar{D}$ (green triangle). The surface nodal line ends at the crossing point of the two projected nodal rings marked by a green arrow in Fig. $4 a$.

To have a better view of the doubly degenerate surface states along the $\bar{B}-\bar{D}$ high-symmetry line, we plot the isofrequency surface arcs for different phonon frequencies in Fig. 4b-e. As shown in Fig. 4b, at the exact frequency of the doubly degenerate projected bulk band crossings (green arrow) along $\bar{B}-\bar{D}$ at $6.410 \mathrm{THz}$, the two surface arcs (cyan triangle) merge at the projected bulk band crossings. For a phonon frequency of $6.414 \mathrm{THz}$, the two surface arcs (cyan triangle) move towards each other, forming two arc crossing points (green triangle) along $\bar{B}-\bar{D}$ in Fig. 4c. At $6.415 \mathrm{THz}$, the two arc crossing points move to the $\bar{D}$ point until they merge into a single arc crossing point [green triangle in Fig. 4d]. Further increasing the phonon frequency eventually leads to two separate surface arcs as the two arcs no longer touch [navy triangle in Fig. 4f]. The arc crossing points on the isofrequency plane belong to the doubly degenerate surface nodal line in the frequency-momentum space [green triangle in Fig. 4a].

The surface nodal line in SnIP is protected by the combination of time-reversal symmetry and glide mirror symmetry $\vartheta=\mathcal{T} \bar{M}_{y}$. For systems with time-reversal symmetry $\mathcal{T}$ and any nonsymmorphic spatial symmetry $G$, the 'Kramers-like' degenerate nodal line appears when $(\mathcal{T} G)^{2}=-1$, either along the $\mathcal{T} G$ invariant lines or on the $\mathcal{T} G$ invariant planes, and a degenerate line can thus be protected by the $\mathcal{T}$ G symmetry. This applies to line degeneracies in both the bulk states $^{93}$ and the surface states ${ }^{53-55}$. SnIP has glide mirror symmetry $\bar{M}_{y}=\left\{M_{y} \mid 00 \frac{1}{2}\right\}$, and $\left(\mathcal{T} \bar{M}_{y}\right)^{2}=\mathrm{e}^{\mathrm{i} 2 \pi q_{z}}\left(q_{z}\right.$ in units of the reciprocal lattice vector $2 \pi / c$ ). On the (100) surface, both $q_{z}=0$ and $q_{z}=0.5$ are $\mathcal{T} \bar{M}_{y}$ invariant lines, and $\left(\mathcal{T} \bar{M}_{y}\right)^{2}=-1$ only occurs when $q_{z}=0.5$, corresponding to the $\bar{B}-\bar{D}$ high-symmetry line. As a result, the doubly degenerate surface nodal line along $\bar{B}-\bar{D}$ is protected by the $\mathcal{T} \bar{M}_{y}$ symmetry.

If we change the surface termination (denoted as surface 2) along the (100) direction, the doubly degenerate surface states along the $\bar{B}-\bar{D}$ high-symmetry line in Fig. $4 \mathrm{f}$ no longer exist in the frequency range $6.410-6.415 \mathrm{THz}$ but move to the lower frequency range $6.384-6.410 \mathrm{THz}$. The distribution of the surface nodal line changes to the other side of $\bar{B}-\bar{D}$, from the purple shaded area in Fig. 5a to that in Fig. 5b.

To explain the alternation between the distributions of the surface nodal line, we need to understand how $\mathcal{T} \bar{M}_{y}$ influences the Zak phase on the (100) surface Brillouin zone. The Zak phase provides information on the distribution of the doubly degenerate surface states on the (100) surface, and can be integrated along the (100) direction,

$\gamma\left(q_{b}, q_{c}\right)=\mathrm{i} \int_{-\pi}^{\pi} \sum_{v=\text { occu. }}\left\langle\psi(\mathbf{q} v)\left|\frac{\partial}{\partial q_{a}}\right| \psi(\mathbf{q} v)\right\rangle d q_{a}$,

where $\psi(\mathbf{q} v)$ is the phonon eigenvector with wave vector $\mathbf{q}$ and band index $v$. Note that on the (100) surface Brillouin zone, $q_{b} \| q_{y}$ and $q_{c} \| q_{z}$, therefore we use $\left(q_{x}, q_{y}, q_{z}\right)$ instead of $\left(q_{a}, q_{b}, q_{c}\right)$ to be consistent with $\mathcal{T} \bar{M}_{y}$. When $q_{z}=0.5,\left(\mathcal{T} \bar{M}_{y}\right)^{2}=-1$ and $\psi_{1}\left(-q_{x}, q_{y}, 0.5\right)=\left(\mathcal{T} \bar{M}_{y}\right) \cdot \psi_{2}\left(q_{x}, q_{y}, 0.5\right)$. Therefore, we can classify the phonon states at constant $q_{y}$ and $q_{z}=0.5$ into two subgroups $\left\{\psi_{1}(\mathbf{q})\right\}$ and $\left\{\psi_{2}(\mathbf{q})\right\}$, i.e. $\left\{\psi_{1}(\mathbf{q})\right\}$ and $\left\{\psi_{2}(\mathbf{q})\right\}$ are $\mathcal{T} \bar{M}_{y}$ related. Therefore, for the $\bar{B}-\bar{D}$ high-symmetry line $\left(q_{z}=0.5\right)$ on the (100) surface Brillouin zone, the Zak phases for the two subgroups $\left\{\psi_{1}(\mathbf{q})\right\}$ and $\left\{\psi_{2}(\mathbf{q})\right\}$ are equal to each other, i.e. $\gamma_{1}\left(q_{y}, 0.5\right)=\gamma_{2}\left(q_{y}, 0.5\right)$. Due to $\mathcal{P} \mathcal{T}, \gamma_{1}\left(q_{y}, 0.5\right)=\gamma_{2}\left(q_{y}, 0.5\right)$ is either 0 or $\pi$, which depends on the surface termination (i.e. choice of the inversion centre). When $\gamma_{1}\left(q_{y}\right.$, $0.5)=\gamma_{2}\left(q_{y}, 0.5\right)=0$, there are no surface states as the total Zak 
(a)
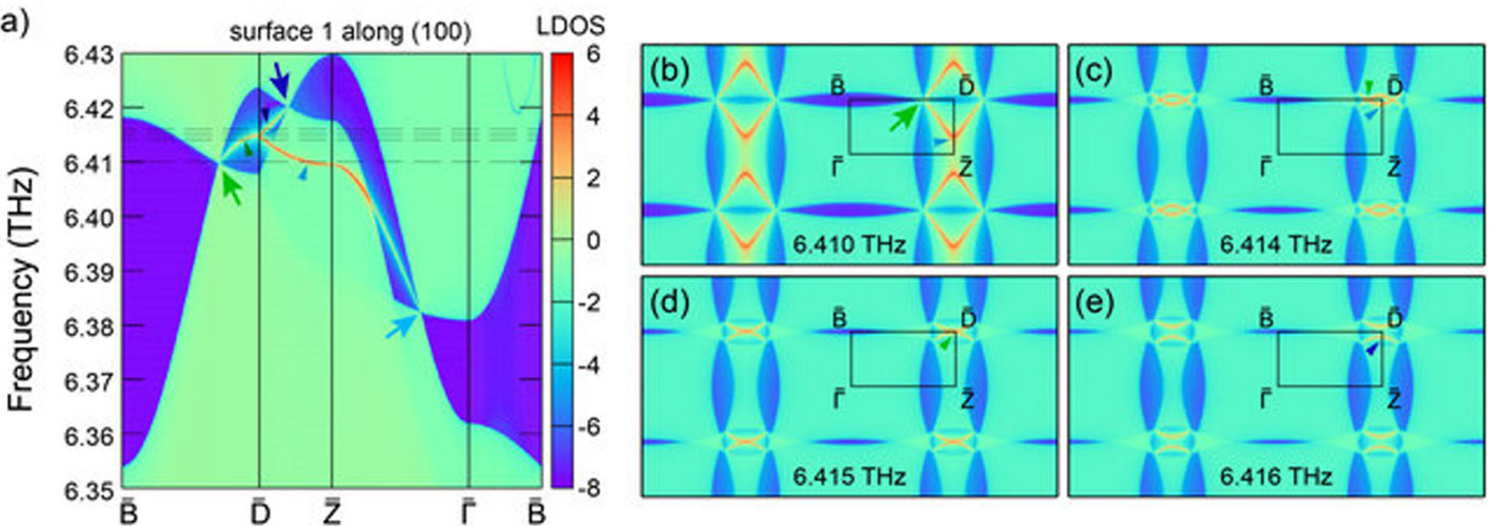

(f)
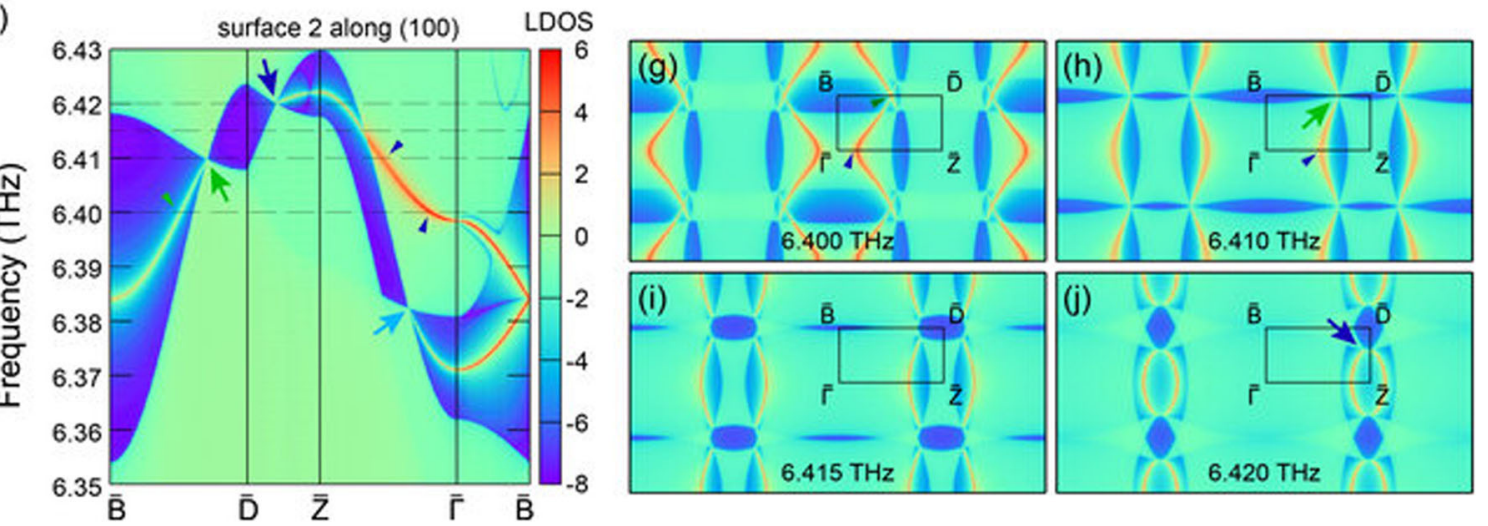

Fig. 4 Topological surface states of SnIP. a Topological surface states of surface 1 along the (100) direction, as well as the surface arcs for phonon frequencies of $\mathbf{b} 6.410 \mathrm{THz}, \mathbf{c} 6.414 \mathrm{THz}, \mathbf{d} 6.415 \mathrm{THz}$ and e $6.416 \mathrm{THz}$. f Topological surface states of surface 2 along the (100) direction, as well as the surface arcs for phonon frequencies of $\mathbf{g} 6.400 \mathrm{THz}, \mathbf{h} 6.410 \mathrm{THz}, \mathbf{i} 6.415 \mathrm{THz}$ and $\mathbf{j} 6.420 \mathrm{THz}$.

phase $\gamma=\gamma_{1}+\gamma_{2}=0$. When $\gamma_{1}\left(q_{y,}, 0.5\right)=\gamma_{2}\left(q_{y,}, 0.5\right)=\pi$, the total Zak phase $\gamma=2 \pi$, leading to doubly degenerate surface states. For surface 1, the purple shaded line in Fig. 5a has a total Zak phase of $2 \pi$ with two surface states distributed in this area, while the green shaded area corresponds to $\gamma=0$ and there are no surface states in this area. For surface 2, the Zak phase of the purple shaded area and the colourless area swap, as shown in Fig. 5b, and the distribution of the surface nodal line switches to the new purple shaded area. It should be noticed that the standard definition of Zak phase is in terms of modulo $2 \pi$, and a Zak phase of $2 \pi$ is therefore equal to zero under this definition. However, in our case, a total Zak phase of $2 \pi$ corresponds to $\gamma_{1}=\gamma_{2}=\pi$, i.e. two $\pi$ Zak phase within each subgroup, indicating two surface states emerging from each subgroup. On the other hand, a total Zak phase of zero corresponds to two zero Zak phase within each group, leading to vanishing surface states.

It is worth mentioning that bending SnIP rods does not break the time-reversal and glide mirror symmetries on the (100) surface of SnIP. Therefore, the degenerate topological surface states remain unchanged under bending.

\section{DISCUSSION}

In terms of fundamental physics and applications, topological bulk phonons in double-helical SnIP may lead to non-dissipative surface phonons, which offer a promising platform for designing thermal devices. The existence of surface nodal lines also provides opportunities to study surface transport properties for thermoelectric devices. As another example, the bulk and surface nodal lines/rings in the phonon spectra result in large phonon DOS, which may also enhance surface superconductivity. Additionally, because of its quasi-one-dimensional crystal structure, SnIP could also be a promising material platform to investigate one-dimensional physical models such as the Su-Schrieffer-Heeger (SSH) model ${ }^{83}$ and Majorana quantum wires ${ }^{84,85}$. With its high mechanical flexibility, it is also interesting to investigate the interplay between flexoelectricity and topology. Our work provides a starting point to explore all these phenomena and applications.

In summary, we find that double-helical SnIP exhibits nodal-ring phonons in the bulk and nodal-line phonons on the surface based on first-principles calculations and group theory analysis. Benefiting from a quasi-one-dimensional crystal structure, the lattice vibrational modes of the intrachain phosphorus atoms along the helices are highly dispersive. Due to the winding of the helix $P$ chain, these phonon bands are folded at the Brillouin zone boundary, forming single nodal rings across the whole Brillouin zone. The overlap between the two neighbouring nodal rings on the (100) surface leads to a surface nodal line that is protected by the combination of time-reversal and glide mirror symmetries $\mathcal{T} \bar{M}_{y}$. We also propose that different surface terminations result in different distributions of the surface nodal line, which can be understood by different Zak phases in the presence of $\mathcal{T} \bar{M}_{y}$. We demonstrate that, similar to the bulk nodal lines, the surface nodal lines can widely exist when protected by extra symmetries. Our discoveries may offer opportunities to study the degeneracy of the topological surface states and their transport properties.

\section{METHODS}

\section{Density functional theory calculations}

All density functional theory (DFT) calculations are performed using the Vienna ab initio simulation package (vasp) ${ }^{94,95}$. The generalised gradient 
(a)

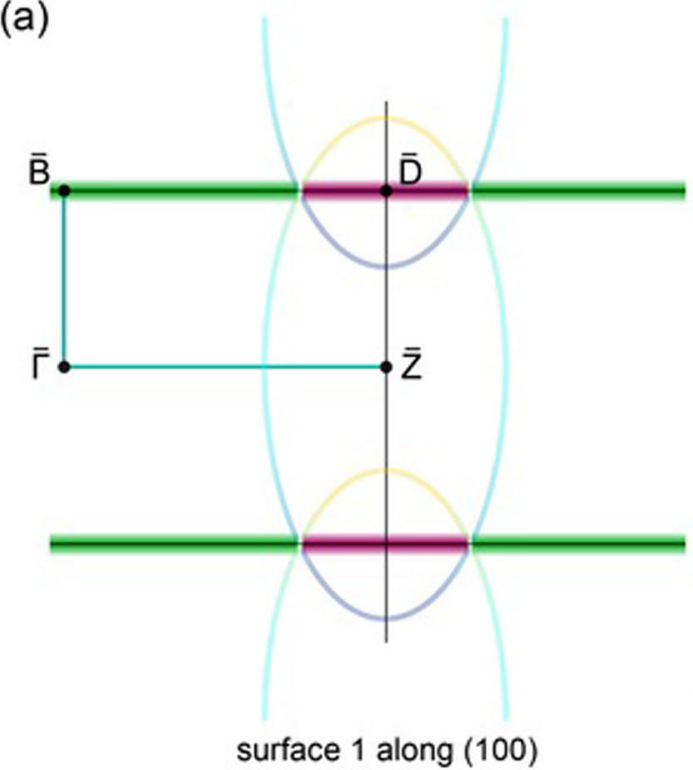

(b)

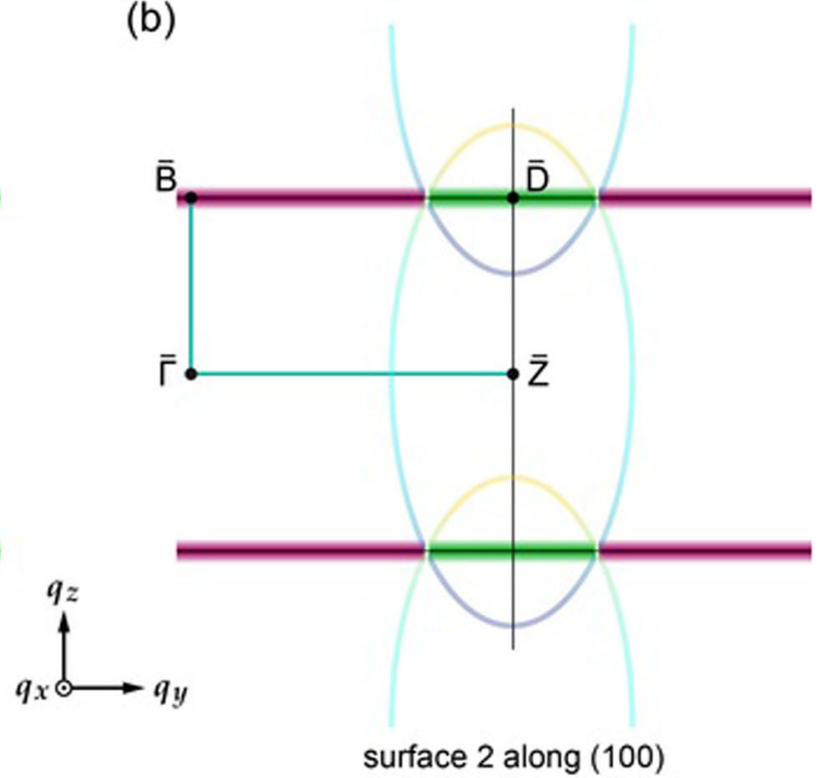

Fig. 5 Zak phase analysis. Zak phase of a surface 1 and $\mathbf{b}$ surface 2 along the (100) direction. The purple and green shaded areas correspond to the total Zak phase $\gamma=2 \pi$ and $\gamma=0$, respectively.

approximation (GGA) is used in the Perdew-Burke-Ernzerhof (PBE) parametrization for the exchange-correlation functional with 14 valence electrons $\left(4 d^{10} 5 s^{2} 5 p^{2}\right)$ for $\mathrm{Sn}, 7$ valence electrons $\left(5 s^{2} 5 p^{5}\right)$ for I and 5 valence electrons $\left(3 s^{2} 3 p^{3}\right)$ for $\mathrm{P}$. We use a plane-wave basis with a kinetic energy cutoff of $600 \mathrm{eV}$ and a $7 \times 5 \times 3 \mathbf{k}$-mesh for structural relaxation until the energy difference is lower than $10^{-6} \mathrm{eV}$ and the Hellman-Feynman force difference is lower than $10^{-4} \mathrm{eV} \AA^{-1}$. The D2 method of Grimme is used to describe the van der Waals interactions between the double helices ${ }^{96}$.

\section{Lattice dynamics properties}

The Hessian matrix and phonon frequencies are calculated based on the finite difference method using a $2 \times 2 \times 1$ supercell with a $3 \times 3 \times 3 \mathbf{k}$-mesh. The phonon dispersion is obtained with the phonopy code ${ }^{97,98}$. The convergence of the supercell is checked by comparing the $2 \times 2 \times 1$ and $1 \times 1 \times 2$ supercells in Supplementary Note 1 of the Supplementary Information. The Born effective charges are also computed using a perturbative approach to account for the splitting between the longitudinal and transverse optical phonon modes (LO-TO splitting) near the Brillouin zone centre ${ }^{99}$, and we find that the LO-TO splitting has a minor influence on the topological properties of phonons because the topological features are far away from the zone centre.

\section{Topological properties}

To obtain the topological properties of the lattice vibrational modes, we use wannierTools to calculate the distribution of the band crossing points/ lines in the whole Brillouin zone ${ }^{92}$. The phonon surface states on the (100) surface are computed using the surface Green's function.

\section{DATA AVAILABILITY}

The datasets generated during and/or analysed during the current study are available from the corresponding authors on reasonable request.

\section{CODE AVAILABILITY}

The related codes are available from the corresponding authors on reasonable request.

Received: 1 June 2021; Accepted: 6 November 2021; Published online: 03 December 2021

\section{REFERENCES}

1. Herring, C. Accidental degeneracy in the energy bands of crystals. Phys. Rev. 52, 365-373 (1937).

2. Heikkilä, T. T., Kopnin, N. B. \& Volovik, G. E. Flat bands in topological media. JETP Lett. 94, 233 (2011).

3. Weng, H., Dai, X. \& Fang, Z. Topological semimetals predicted from first-principles calculations. J. Phys. Condens. Matter 28, 303001 (2016).

4. Bernevig, A., Weng, H., Fang, Z. \& Dai, X. Recent progress in the study of topological semimetals. J. Phys. Soc. Jpn. 87, 041001 (2018).

5. Stenull, O., Kane, C. L. \& Lubensky, T. C. Topological phonons and Weyl lines in three dimensions. Phys. Rev. Lett. 117, 068001 (2016).

6. Liu, Y., Xu, Y., Zhang, S.-C. \& Duan, W. Model for topological phononics and phonon diode. Phys. Rev. B 96, 064106 (2017).

7. He, H. et al. Topological negative refraction of surface acoustic waves in a Weyl phononic crystal. Nature 560, 61-64 (2018).

8. Jia, H. et al. Observation of chiral zero mode in inhomogeneous threedimensional Weyl metamaterials. Science 363, 148 (2019).

9. Li, F.-Y. et al. Weyl magnons in breathing pyrochlore antiferromagnets. Nat. Commun. 7, 12691- (2016).

10. Wu, F., Lovorn, T. \& MacDonald, A. H. Topological exciton bands in Moiré heterojunctions. Phys. Rev. Lett. 118, 147401 (2017).

11. Xu, S.-Y. et al. Discovery of a Weyl Fermion semimetal and topological Fermi arcs. Science 349, 613-617 (2015).

12. Lv, B. Q. et al. Experimental discovery of Weyl semimetal TaAs. Phys. Rev. X 5, 031013 (2015).

13. Weng, H., Fang, C., Fang, Z., Bernevig, B. A. \& Dai, X. Weyl semimetal phase in noncentrosymmetric transition-metal monophosphides. Phys. Rev. X 5, 011029 (2015).

14. Yang, L. X. et al. Weyl semimetal phase in the non-centrosymmetric compound TaAs. Nat. Phys. 11, 728-732 (2015).

15. Novoselov, K. S. et al. Electric field effect in atomically thin carbon films. Science 306, 666 (2004).

16. Zhang, Y., Tan, Y.-W., Stormer, H. L. \& Kim, P. Experimental observation of the quantum hall effect and Berry's phase in graphene. Nature 438, 201-204 (2005).

17. Wang, Z. et al. Dirac semimetal and topological phase transitions in $A_{3} \mathrm{Bi}(A=\mathrm{Na}$, K, Rb). Phys. Rev. B 85, 195320 (2012).

18. Liu, Z. K. et al. Discovery of a three-dimensional topological Dirac semimetal, $\mathrm{Na}_{3} \mathrm{Bi}$. Science 343, 864 (2014).

19. Armitage, N. P., Mele, E. J. \& Vishwanath, A. Weyl and Dirac semimetals in threedimensional solids. Rev. Mod. Phys. 90, 015001 (2018).

20. Peng, B., Yue, C., Zhang, H., Fang, Z. \& Weng, H. Predicting Dirac semimetals based on sodium ternary compounds. npj Comput. Mater. 4, 68 (2018).

21. Zhang, T. et al. Double-Weyl phonons in transition-metal monosilicides. Phys. Rev. Lett. 120, 016401 (2018). 
22. Li, J. et al. Computation and data driven discovery of topological phononic materials. Nat. Commun. 12, 1204- (2021).

23. Weng, $H$. et al. Topological node-line semimetal in three-dimensional graphene networks. Phys. Rev. B 92, 045108 (2015).

24. Fang, C., Chen, Y., Kee, H.-Y. \& Fu, L. Topological nodal line semimetals with and without spin-orbital coupling. Phys. Rev. B 92, 081201 (2015).

25. Yu, R., Weng, H., Fang, Z., Dai, X. \& Hu, X. Topological node-line semimetal and Dirac semimetal state in antiperovskite $\mathrm{Cu}_{3} \mathrm{PdN}$. Phys. Rev. Lett. 115, 036807 (2015).

26. Bzdusek, T., Wu, Q., Ruegg, A., Sigrist, M. \& Soluyanov, A. A. Nodal-chain metals. Nature 538, 75-78 (2016).

27. Huang, H., Liu, J., Vanderbilt, D. \& Duan, W. Topological nodal-line semimetals in alkaline-earth stannides, germanides, and silicides. Phys. Rev. B 93, 201114 (2016).

28. $\mathrm{Hu}$, J. et al. Evidence of topological nodal-line Fermions in $\mathrm{ZrSiSe}$ and $\mathrm{ZrSiTe}$. Phys. Rev. Lett. 117, 016602 (2016).

29. Bian, G. et al. Topological nodal-line Fermions in spin-orbit metal $\mathrm{PbTaSe}_{2}$. Nat. Commun. 7, 10556 (2016).

30. Yang, S.-Y. et al. Symmetry demanded topological nodal-line materials. Adv. Phys.: X 3, 1414631 (2018).

31. Lian, J. et al. Multi-loop node line states in ternary MgSrSi-type crystals. npj Comput. Mater. 5, 10 (2019).

32. Fang, C., Weng, H., Dai, X. \& Fang, Z. Topological nodal line semimetals. Chin. Phys. B 25, 117106 (2016).

33. Peng, B., Bravić, I., MacManus-Driscoll, J. L. \& Monserrat, B. Topological semimetallic phase in $\mathrm{PbO}_{2}$ promoted by temperature. Phys. Rev. B 100, 161101 (2019).

34. Zhang, T. et al. Catalogue of topological electronic materials. Nature 566 , 475-479 (2019).

35. Tang, F., Po, H. C., Vishwanath, A. \& Wan, X. Comprehensive search for topological materials using symmetry indicators. Nature 566, 486-489 (2019).

36. Vergniory, M. G. et al. A complete catalogue of high-quality topological materials. Nature 566, 480-485 (2019).

37. Son, D. T. \& Spivak, B. Z. Chiral anomaly and classical negative magnetoresistance of Weyl metals. Phys. Rev. B 88, 104412 (2013).

38. Xiong, J. et al. Evidence for the chiral anomaly in the Dirac semimetal $\mathrm{Na}_{3} \mathrm{Bi}$. Science 350, 413 (2015).

39. Belopolski, I. et al. Discovery of topological Weyl Fermion lines and drumhead surface states in a room temperature magnet. Science 365, 1278 (2019).

40. Bouhon, A. et al. Non-abelian reciprocal braiding of Weyl points and its manifestation in ZrTe. Nat. Phys. 16, 1137-1143 (2020).

41. Sun, Y., Wu, S.-C. \& Yan, B. Topological surface states and Fermi arcs of the noncentrosymmetric Weyl semimetals TaAs, TaP, NbAs, and NbP. Phys. Rev. B 92, 115428 (2015).

42. Huang, S.-M. et al. A Weyl Fermion semimetal with surface Fermi arcs in the transition metal monopnictide TaAs class. Nat. Commun. 6, 7373 (2015).

43. Deng, K. et al. Experimental observation of topological Fermi arcs in type-ii Weyl semimetal MoTe 2 . Nat. Phys. 12, 1105 (2016).

44. Tamai, A. et al. Fermi arcs and their topological character in the candidate type-ii Weyl semimetal MoTe 2 . Phys. Rev. X 6, 031021 (2016).

45. Burkov, A. A., Hook, M. D. \& Balents, L. Topological nodal semimetals. Phys. Rev. $B$ 84, 235126 (2011).

46. Okugawa, R. \& Murakami, S. Dispersion of Fermi arcs in Weyl semimetals and their evolutions to Dirac cones. Phys. Rev. B 89, 235315 (2014).

47. Fang, C., Lu, L., Liu, J. \& Fu, L. Topological semimetals with helicoid surface states. Nat. Phys. 12, 936-941 (2016)

48. Fu, L., Kane, C. L. \& Mele, E. J. Topological insulators in three dimensions. Phys. Rev. Lett. 98, 106803 (2007).

49. Zhang, $\mathrm{H}$. et al. Topological insulators in $\mathrm{Bi}_{2} \mathrm{Se}_{3}, \mathrm{Bi}_{2} \mathrm{Te}_{3}$ and $\mathrm{Sb}_{2} \mathrm{Te}_{3}$ with a single Dirac cone on the surface. Nat. Phys. 5, 438-442 (2009).

50. Chen, Y. L. et al. Experimental realization of a three-dimensional topological insulator, $\mathrm{Bi}_{2} \mathrm{Te}_{3}$. Science 325, 178 (2009).

51. Dziawa, P. et al. Topological crystalline insulator states in $\mathrm{Pb}_{1-x} \mathrm{Sn}_{x} \mathrm{Se}$. Nat. Mater. 11, 1023 (2012).

52. Wojek, B. M. et al. Direct observation and temperature control of the surface Dirac gap in a topological crystalline insulator. Nat. Commun. 6, 8463- (2015).

53. Wang, Z., Alexandradinata, A., Cava, R. J. \& Bernevig, B. A. Hourglass Fermions. Nature 532, 189 (2016).

54. Alexandradinata, A., Wang, Z. \& Bernevig, B. A. Topological insulators from group cohomology. Phys. Rev. X 6, 021008 (2016).

55. Ezawa, M. Hourglass Fermion surface states in stacked topological insulators with nonsymmorphic symmetry. Phys. Rev. B 94, 155148 (2016).

56. Kuo, C.-S., Chang, T.-R., Xu, S.-Y. \& Jeng, H.-T. Unconventional topological phase transition in non-symmorphic material $\mathrm{KHgX}(\mathrm{X}=\mathrm{As}, \mathrm{Sb}, \mathrm{Bi})$. npj Comput. Mater. 5, 65 (2019).

57. Hosen, M. M. et al. Distinct multiple Fermionic states in a single topological metal. Nat. Commun. 9, 3002- (2018).
58. Cheng, H., Sha, Y., Liu, R., Fang, C. \& Lu, L. Discovering topological surface states of Dirac points. Phys. Rev. Lett. 124, 104301 (2020).

59. Cai, X. et al. Symmetry-enforced three-dimensional Dirac phononic crystals. Light Sci. Appl. 9, 38- (2020).

60. Miao, H. et al. Observation of double Weyl phonons in parity-breaking FeSi. Phys. Rev. Lett. 121, 035302 (2018).

61. Li, J. et al. Coexistent three-component and two-component Weyl phonons in TiS, ZrSe, and HfTe. Phys. Rev. B 97, 054305 (2018).

62. Xia, B. W., Wang, R., Chen, Z. J., Zhao, Y. J. \& Xu, H. Symmetry-protected ideal typeii Weyl phonons in CdTe. Phys. Rev. Lett. 123, 065501 (2019).

63. Zhang, T. T. et al. Phononic helical nodal lines with $\mathcal{P} \mathcal{T}$ protection in $\mathrm{MoB}_{2}$. Phys. Rev. Lett. 123, 245302 (2019).

64. Liu, Y., Chen, X. \& Xu, Y. Topological phononics: from fundamental models to real materials. Adv. Funct. Mater. 30, 1904784 (2020).

65. Peng, B., Hu, Y., Murakami, S., Zhang, T. \& Monserrat, B. Topological phonons in oxide perovskites controlled by light. Sci. Adv. 6, eabd1618 (2020).

66. Liu, Q.-B., Qian, Y., Fu, H.-H. \& Wang, Z. Symmetry-enforced Weyl phonons. npj Comput. Mater. 6, 95 (2020).

67. Wang, Z., Zhou, W., Rudenko, A. N. \& Yuan, S. Lattice dynamics and topological surface phonon states in cuprous oxide $\mathrm{Cu}_{2}$ O. Phys. Rev. B 103, 195137 (2021).

68. Tang, D.-S. \& Cao, B.-Y. Topological effects of phonons in GaN and AlGaN: a potential perspective for tuning phonon transport. J. Appl. Phys. 129, 085102 (2021).

69. Liu, Q.-B., Fu, H.-H. \& Wu, R. Topological phononic nodal hexahedron net and nodal links in the high-pressure phase of the semiconductor $\mathrm{CuCl}$. Phys. Rev. $B$ 104, 045409 (2021)

70. Liu, P.-F. et al. First-principles prediction of ideal type-ii Weyl phonons in wurtzite ZnSe. Phys. Rev. B 103, 094306 (2021).

71. Liu, Q.-B., Wang, Z.-Q. \& Fu, H.-H. Ideal topological nodal-surface phonons in RbTeAu-family materials. Phys. Rev. B 104, L041405 (2021).

72. You, J.-Y., Sheng, X.-L. \& Su, G. Topological gimbal phonons in t-carbon. Phys. Rev. $B$ 103, 165143 (2021).

73. Xie, $C$. et al. Sixfold degenerate nodal-point phonons: Symmetry analysis and materials realization. Phys. Rev. B 104, 045148 (2021).

74. Zheng, B., Zhan, F., Wu, X., Wang, R. \& Fan, J. Hourglass phonons jointly protected by symmorphic and nonsymmorphic symmetries. Phys. Rev. B 104, L060301 (2021).

75. Wang, J. et al. Coexistence of zero-, one-, and two-dimensional degeneracy in tetragonal $\mathrm{SnO}_{2}$ phonons. Phys. Rev. B 104, L041107 (2021).

76. Peng, B., Bouhon, A., Monserrat, B. \& Slager, R.-J. Non-abelian braiding of phonons in layered silicates. Preprint at arXiv: 2105.08733 (2021).

77. Pfister, D. et al. Inorganic double helices in semiconducting SnIP. Adv. Mater. 28 9783-9791 (2016).

78. Saleh, L. M. A., Dziedzic, R. \& Spokoyny, A. M. An inorganic twist in nanomaterials: making an atomically precise double helix. ACS Cent. Sci. 2, 685-686 (2016).

79. Utrap, A., Xiang, N. Y. \& Nilges, T. A yield-optimized access to double-helical SnIP via a Sn/Snl 2 approach. J. Cryst. Growth 475, 341-345 (2017).

80. $\mathrm{Li}, \mathrm{X}$. et al. Landscape of DNA-like inorganic metal free double helical semiconductors and potential applications in photocatalytic water splitting. J. Mater. Chem. A 5, 8484-8492 (2017).

81. Ott, C. et al. Flexible and ultrasoft inorganic 1d semiconductor and heterostructure systems based on SnIP. Adv. Funct. Mater. 29, 1900233 (2019).

82. Baumgartner, M., Weihrich, R. \& Nilges, T. Inorganic SnIP-type double helices in main-group chemistry. Chem. Eur. J. 23, 6452-6457 (2017).

83. Su, W. P., Schrieffer, J. R. \& Heeger, A. J. Soliton excitations in polyacetylene. Phys. Rev. B 22, 2099-2111 (1980).

84. Kitaev, A. Y. Unpaired majorana Fermions in quantum wires. Phys. -Uspekhi 44, 131-136- (2001).

85. Franz, M. Majorana's wires. Nat. Nanotechnol. 8, 149-152 (2013).

86. Müller, U. Die symmetrie von Spiralketten. Acta Crystallogr. B 73, 443-452 (2017)

87. Po, H. C., Vishwanath, A. \& Watanabe, H. Symmetry-based indicators of band topology in the 230 space groups. Nat. Commun. 8, 50- (2017).

88. Song, Z., Zhang, T. \& Fang, C. Diagnosis for nonmagnetic topological semimetals in the absence of spin-orbital coupling. Phys. Rev. X 8, 031069 (2018).

89. Kruthoff, J., de Boer, J., van Wezel, J., Kane, C. L. \& Slager, R.-J. Topological classification of crystalline insulators through band structure combinatorics. Phys. Rev. X 7, 041069 (2017)

90. Zhang, T. et al. Diagnosis scheme for topological degeneracies crossing highsymmetry lines. Phys. Rev. Res. 2, 022066 (2020).

91. Zhang, T. \& Murakami, S. Predicting topological materials: symmetry-based indicator theories and beyond. J. Phys. D Appl. Phys. 54, 414002 (2021).

92. Wu, Q., Zhang, S., Song, H.-F., Troyer, M. \& Soluyanov, A. A. WannierTools: an open-source software package for novel topological materials. Comput. Phys. Commun. 224, 405-416 (2018). 
93. Takahashi, R., Hirayama, M. \& Murakami, S. Spinless hourglass nodal-line semimetals. Phys. Rev. B 96, 155206 (2017).

94. Kresse, G. \& Furthmüller, J. Efficient iterative schemes for ab initio total-energy calculations using a plane-wave basis set. Phys. Rev. B 54, 11169-11186 (1996).

95. Kresse, G. \& Furthmüller, J. Efficiency of ab-initio total energy calculations for metals and semiconductors using a plane-wave basis set. Comput. Mater. Sci. 6 , 15 - 50 (1996).

96. Grimme, S. Semiempirical GGA-type density functional constructed with a longrange dispersion correction. J. Comput. Chem. 27, 1787-1799 (2006).

97. Togo, A., Oba, F. \& Tanaka, I. First-principles calculations of the ferroelastic transition between rutile-type and $\mathrm{CaCl}_{2}$-type $\mathrm{SiO}_{2}$ at high pressures. Phys. Rev. $B$ 78, 134106 (2008).

98. Togo, A. \& Tanaka, I. First principles phonon calculations in materials science. Scr. Mater. 108, 1-5 (2015).

99. Gajdoš, M., Hummer, K., Kresse, G., Furthmüller, J. \& Bechstedt, F. Linear optical properties in the projector-augmented wave methodology. Phys. Rev. B 73, 045112 (2006).

\section{ACKNOWLEDGEMENTS}

B.P. and B.M. acknowledge support from the Winton Programme for the Physics of Sustainability, and B.M. also acknowledges support from the Gianna Angelopoulos Programme for Science, Technology, and Innovation. S.M. and T.Z. acknowledge the supports from JSPS KAKENHI Grants No. JP18H03678 and No. JP2OH04633, Tokodai Institute for Element Strategy (TIES) funded by MEXT Elements Strategy Initiative to Form Core Research Center. T.Z. also acknowledges the support by the Japan Society for the Promotion of Science (JSPS), KAKENHI Grant No. JP21K13865. The calculations are performed using resources provided by the Cambridge Tier- 2 system operated by the University of Cambridge Research Computing Service (http://www.hpc.cam.ac.uk) and funded by EPSRC Tier-2 capital grant EP/P020259/1, and also with computational support from the UK Materials and Molecular Modelling Hub, which is partially funded by EPSRC (EP/P020194), for which access is obtained via the UKCP consortium and funded by EPSRC grant EP/P022561/1

\section{AUTHOR CONTRIBUTIONS}

B.P., B.M. and T.Z. devised the project idea and prepared the main part of the manuscript. B.P. performed the first-principles calculations. S.M. and T.Z. analysed the topological features. B.P., S.M., B.M. and T.Z. discussed the results and the ideas for analysis and edited the manuscript.

\section{COMPETING INTERESTS}

The authors declare no competing interests.

\section{ADDITIONAL INFORMATION}

Supplementary information The online version contains supplementary material available at https://doi.org/10.1038/s41524-021-00667-6.

Correspondence and requests for materials should be addressed to Bartomeu Monserrat or Tiantian Zhang.

Reprints and permission information is available at http://www.nature.com/ reprints

Publisher's note Springer Nature remains neutral with regard to jurisdictional claims in published maps and institutional affiliations.

(i) Open Access This article is licensed under a Creative Commons c. Attribution 4.0 International License, which permits use, sharing, adaptation, distribution and reproduction in any medium or format, as long as you give appropriate credit to the original author(s) and the source, provide a link to the Creative Commons license, and indicate if changes were made. The images or other third party material in this article are included in the article's Creative Commons license, unless indicated otherwise in a credit line to the material. If material is not included in the article's Creative Commons license and your intended use is not permitted by statutory regulation or exceeds the permitted use, you will need to obtain permission directly from the copyright holder. To view a copy of this license, visit http://creativecommons. org/licenses/by/4.0/.

(c) The Author(s) 2021 